\title{
Hypoxic Exposure Increases Energy Expenditure by Increasing Carbohydrate Oxidation in Mice
}

\author{
Yeram Park $\mathbb{D}^{\mathbb{D}},{ }^{1}$ Deunsol Hwang, ${ }^{1}$ Hun-Young Park, ${ }^{2,3}$ Jisu Kim, ${ }^{2,3}$ and Kiwon Lim $\mathbb{D}^{1,2,3}$ \\ ${ }^{1}$ Department of Physical Education in Graduated School, Konkuk University, Seoul, Republic of Korea \\ ${ }^{2}$ Department of Sports Medicine and Science in Graduated School, Konkuk University, Seoul, Republic of Korea \\ ${ }^{3}$ Physical Activity and Performance Institute, Konkuk University, Seoul, Republic of Korea \\ Correspondence should be addressed to Kiwon Lim; exercise@konkuk.ac.kr
}

Received 15 January 2020; Accepted 11 May 2020; Published 24 June 2020

Academic Editor: Yun-Peng Chao

Copyright (c) 2020 Yeram Park et al. This is an open access article distributed under the Creative Commons Attribution License, which permits unrestricted use, distribution, and reproduction in any medium, provided the original work is properly cited.

\begin{abstract}
Aims. Hypoxic exposure improves glucose metabolism. We investigated to validate the hypothesis that carbohydrate (CHO) oxidation could increase in mice exposed to severe hypoxic conditions. Methods. Seven-week-old male ICR mice $(n=16)$ were randomly divided into two groups: the control group $(\mathrm{CON}$ ) was kept in normoxic condition (fraction of inspired $\mathrm{O} 2=21 \%$ ) and the hypoxia group (HYP) was exposed to hypoxic condition (fraction of inspired $\mathrm{O} 2=12 \%, \approx$ altitude of $4,300 \mathrm{~m}$ ). The CON group was pair-fed with the HYP group. After 3 weeks of hypoxic exposure, we measured respiratory metabolism (energy expenditure and substrate utilization) at normoxic conditions for 24 hours using an open-circuit calorimetry system. In addition, we investigated changes in carbohydrate mechanism-related protein expression, including hexokinase 2 (HK2), pyruvate dehydrogenase (PDH), pyruvate dehydrogenase kinase 4 (PDK4), and regulator of the genes involved in energy metabolism (peroxisome proliferator-activated receptor gamma coactivator 1-alpha, PGC1 $\alpha$ ) in soleus muscle. Results. Energy expenditure (EE) and CHO oxidation over 24 hours were higher in the HYP group by approximately $15 \%$ and $34 \%(p<0.001)$, respectively. Fat oxidation was approximately $29 \%$ lower in the HYP group than the CON group $(p<0.01)$. Body weight gains were significantly lower in the HYP group than in the CON group (CON vs. HYP; $1.9 \pm 0.9$ vs. $-0.3 \pm 0.9 ; p<0.001$ ). Hypoxic exposure for 3 weeks significantly reduced body fat by approximately $42 \%(p<0.001)$. PDH and PGC1 $\alpha$ protein levels were significantly higher in the HYP group $(p<0.05)$. Additionally, HK2 was approximately $21 \%$ higher in the HYP group. Conclusions. Hypoxic exposure might significantly enhance $\mathrm{CHO}$ oxidation by increasing the expression of PDH and $\mathrm{HK} 2$. This investigation can be useful for patients with impaired glucose metabolism, such as those with type 2 diabetes.
\end{abstract}

\section{Introduction}

According to the International Society for Mountain Medicine, high altitude is defined as 3,000-5,500 $\mathrm{m}$ above sea level (equivalent fraction of inspired $\mathrm{O} 2=10.9-14.8 \%$ ) and extreme altitude as $>5,500 \mathrm{~m}$ above sea level (equivalent fraction of inspired $\mathrm{O} 2<10.9 \%)$. It is estimated that more than 140 million people live at $2,500 \mathrm{~m}$ above sea level, mainly in North, Central, and South America; Asia; and Eastern Africa [1-3]. High altitude populations have lower blood glucose levels and lower incidence of type 2 diabetes. Hill et al. [4] demonstrated that blood glucose and insulin resistance were lower following gradual ascent in altitude (3,600-5120 m). Other studies have found that prolonged exposure to high altitude $(3,500 \mathrm{~m})$ may decrease blood glucose [5]. Mice, like humans, adapt readily to prolonged hypoxic exposure. Other rodents (e.g., rats) are not found at $>3,000 \mathrm{~m}$, whereas mice (Mus musculus) and deer mice (Peromyscus maniculatus) live as high as 4,500 $\mathrm{m}$ above sea level [6]. Deer mice expend significantly more energy per day than mice living lowland, which would require a high rate of $\mathrm{O} 2$ consumption. These mice may rely on carbohydrates as a substrate because of the relatively high yield of ATP per mole of $\mathrm{O} 2$ consumed in carbohydrate metabolism [7]. Enhanced carbohydrate oxidation following exposure to hypoxia (at 4,300-4,540 $\mathrm{m}$ ) is expected, as previous studies have demonstrated that healthy humans exhibit increased levels of glucose disposal at high altitudes compared to sea level altitudes $[8,9]$. Roberts et al. 


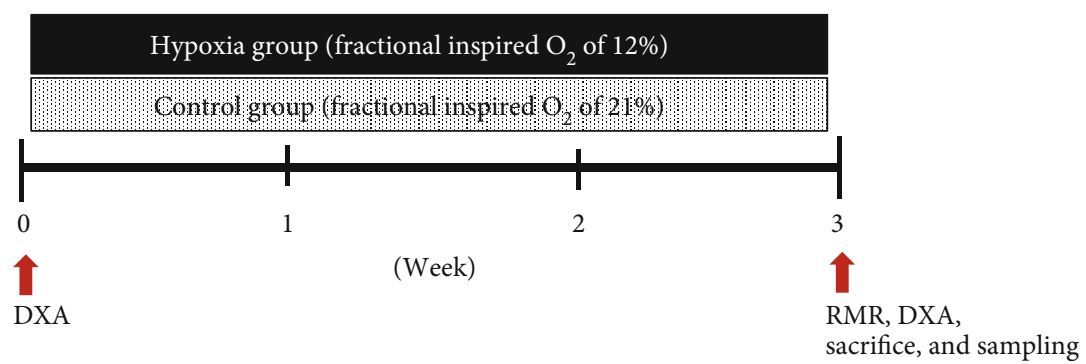

Figure 1: Experiment design.

[10] reported that reliance on fatty acid consumption decreased under conditions of high altitude $(4,300 \mathrm{~m})$. However, in previous studies, whether hypoxic exposure can induce glucose utilization as a substrate substantially remains unclear. In addition, previous studies have not established metabolic responses to hypoxic exposure. The activity of glucose transporter following exposure to hypoxic condition (fractional inspired $\mathrm{O} 2$ of $10 \%$ ) does not change compared with normoxic conditions even though insulin sensitivity was improved [3]. Furthermore, in some cases, although carbohydrate oxidation was augmented in high-altitude groups, blood glucose and insulin concentration were not different with sea level [11]. From these studies, it is clear that the metabolic effect is dependent on the severity and period of the hypoxic exposure. The influence of exposure to hypoxia on metabolic responses is apparent under severe hypoxia (i.e., altitude $\geq 4,000 \mathrm{~m}$ ) and prolonged acclimatization ( $>3$ weeks) $[3,11]$.

Collectively, the studies of carbohydrate metabolism were inconsistent, and the influence of hypoxia on metabolic variables remains unclear. Therefore, it is necessary to confirm carbohydrate metabolism with biomarkers (i.e., enzymes related to carbohydrate mechanism). The aim of the present study was to investigate the influence of hypoxic exposure on carbohydrate metabolism in the whole body. We also explored changes in the expression levels of enzymes related to carbohydrate oxidation (e.g., hexokinase 2 (HK2), pyruvate dehydrogenase $(\mathrm{PDH})$, and pyruvate dehydrogenase kinase 4 (PDK4)) and a regulator of the genes involved in energy metabolism (peroxisome proliferator-activated receptor gamma coactivator 1 -alpha $(\mathrm{PGC1} \alpha)$ ) to elucidate potential mechanisms responsible for any observed changes. We hypothesized that hypoxic exposure could promote carbohydrate oxidation and increase the expression level of enzymes related to carbohydrate metabolism.

\section{Methods}

2.1. Animals. Seven-week-old male ICR mice $(n=16)$ were used for experimentation. Mice were purchased from Orient Bio Inc. (Seongnam, Korea). All mice were housed in standard plastic cages under controlled humidity (50\%) and temperature $\left(23 \pm 1^{\circ} \mathrm{C}\right)$ condition in a $12 \mathrm{~h} / 12 \mathrm{~h}$ dark/light cycle. Mice were acclimated to the laboratory housing condition for 1 week. Mice were divided randomly into two groups: the control group $(\mathrm{CON})$ was kept in normoxia (fraction of inspired $\mathrm{O} 2=21 \%$ ) and the hypoxia group (HYP) was exposed to normobaric hypoxia (fraction of inspired $\mathrm{O} 2=$ $12 \%$, zaltitude $4,300 \mathrm{~m}$ ). The CON group was pair-fed with the HYP group. Mice received a nonpurified commercial diet (65\% carbohydrate, $21 \%$ protein, and $14 \%$ fat) and water ad libitum. The animal experiments were conducted according to protocols reviewed and approved by the Konkuk University Institutional Animal Care and Use Committee (permit number: KU19086).

2.2. Hypoxic Exposure. The HYP group was placed in identical commercially designed chambers (model HCC-550, SFET, Korea). The duration of exposure was 3 weeks. Programmed gas concentrations were circulated into the chamber, and an internal $\mathrm{O} 2$ analyzer measured the $\mathrm{O} 2$ concentration continuously. Deviations from the fixed concentrations were automatically corrected by a computerized system of solenoid valves controlling gas outlets adding either $\mathrm{N} 2$ or $\mathrm{O} 2$. Ambient $\mathrm{CO} 2$ in the chamber was maintained at less than $0.01 \%$, and humidity was maintained at $40-50 \%$ by circulating the gas through a carbon molecular sieve (CMS). Ambient temperature was kept at $23 \pm 1{ }^{\circ} \mathrm{C}$. Immediately after the 3-week exposure, mice were fasted for 4 hours with free access to water. Mice were anesthetized with avertin and measured dual-energy X-ray absorptiometry (DXA); then, soleus muscle and adipose tissues were quickly removed, frozen in liquid nitrogen, and kept at $-80^{\circ} \mathrm{C}$ until analysis. Control animals were exposed to circulating room air (fraction of inspired $\mathrm{O}_{2}=21 \%$ ) and raised in the same space as the HYP group. The experimental design is summarized in Figure 1.

2.3. Assessment of Body Fat Composition. Fat mass (FM) and fat-free mass (FFM) were determined by DXA (model iNSiGHT VET DXA, Osteosys, Korea). The fat tissues were extracted from the subcutaneous fat, epididymal adipose tissue, perirenal adipose tissue, and mesenteric adipose tissue. To verify the accuracy of DXA measurements, all mice were scanned three times without repositioning. Radiography of body fat was displayed by two modes according to lowdensity fat (green color) and high-density fat (red color) [12]

2.4. Measurement of Resting Metabolic Rate. Resting metabolic rate (RMR) was measured using an animal metabolic chamber for 24 hours based on previous studies [13, 14]. Mice were allowed to freely intake food and water. The flow rate within the chamber was set to be at $3 \mathrm{~L} / \mathrm{min}$. Air from each chamber was passed through an acrylic tube of $6 \mathrm{~mm}$ 
in diameter and $3 \mathrm{~m}$ in length and sampled for $15 \mathrm{~s}$ intervals. The $\mathrm{O} 2$ uptake and $\mathrm{CO} 2$ production of mice were measured using a mass analyzer (model RL-600, Alco System, Chiba, Japan; used as a gas analyzer), a switching system (model ANI6-A-S, Alco System, Chiba, Japan), and a gas analyzer. Energy expenditure, respiratory exchange ratio (RER), level of carbohydrate oxidation, and level of fat oxidation were calculated using gas analysis data.

2.5. Western Blot Analysis. The protocol for western blot analysis was based on previous studies [15]. Frozen soleus muscles were homogenized in RIPA Lysis buffer (1 mL RIPA Lysis, $10 \mu \mathrm{L}$ Protease inhibitor, and $10 \mu \mathrm{L}$ Phosphatase inhibitor per sample; EzRIPA Lysis, ATTO biotechnology, Sungnam, Korea). Muscle lysates were mixed with a rotator for $2 \mathrm{~h}$ at $4^{\circ} \mathrm{C}$ and then centrifuged at $13,000 \mathrm{rpm}$ at $4^{\circ} \mathrm{C}$ for $15 \mathrm{~min}$. Equal amounts of proteins were separated by $12 \%$ SDS-PAGE, transferred on to polyvinylidene fluoride (PVDF) membranes (Millipore, Billerica, MA, USA), and blocked with $5 \%$ skim milk powder. Then, the membranes were incubated with rabbit anti-rat primary antibodies for detection of PDK4 (1:1000, an38242), PGC1 $\alpha(1: 1000$, ab54481) or mouse anti-rat $\beta$-actin $(1: 1000$, sc47778), HK2 (1:1000, sc130358), and PDH $(1: 1000, \operatorname{sc} 377092)$ overnight at $4^{\circ} \mathrm{C}$. Membranes were then incubated with the relevant secondary antibodies (PDK4, PGC1 $\alpha$ : anti-rabbit IgG; $\beta$-actin, HK2, PDH: anti-mouse IgG; all from Santa Cruz) for $1 \mathrm{~h}$ at room temperature. Proteins were detected with the enhanced chemiluminescence (ECL) detection system (Amersham Biosciences, Sweden). The optical density of each band was normalized to the optical density of $\beta$-actin.

2.6. Statistics. Data were presented as the mean \pm SD. SPSS 24.0 software (SPSS, Inc., Chicago, IL, USA) was used for statistical analysis. Respiratory gas, energy expenditure, RER, carbohydrate oxidation, and fat oxidation were analyzed by two-way repeated measure analysis of variance. Differences between the groups were analyzed with an unpaired $t$-test. Statistical significance was defined as $p<0.05, p<0.01, p<0.001$.

\section{Results}

3.1. Body Weight Change and Abdominal Adipose Tissue. As shown in Table 1 , body weight was increased in the CON group during the study period (Pre vs. Post; $34.0 \pm 1.5$ vs. $35.9 \pm 1.5 \mathrm{~g})$, whereas no difference was found in the HYP group (Pre vs. Post; $32.9 \pm 1.1$ vs. $32.5 \pm 1.1 \mathrm{~g}$ ). Body weight gains were significantly lower in the HYP group than in the CON group (CON vs. HYP; $1.9 \pm 0.9$ vs. $-0.3 \pm 0.9 \mathrm{~g} ; p<$ $0.001)$. The total weights of the abdominal adipose tissue were significantly lower in the HYP group than in the CON group (total abdominal, epididymal, and perirenal $p<0.01$; mesenteric $p<0.05$ ).

3.2. Distribution of Fat Mass and Fat-Free Mass Measurements by DXA. The effects of hypoxic exposure on body composition, fat mass, and fat-free mass were determined by DXA. The DXA scans of representative mice are presented in Figure 2. The CON group presented with a more high-density fat area than the HYP group posttreatment. As
TABLE 1: Change in body weight and adipose tissue weight in the CON (g) and HYP (g) groups.

\begin{tabular}{lcc}
\hline & CON & HYP \\
\hline Initial body weight & $34.0 \pm 1.5$ & $32.9 \pm 1.1$ \\
Final body weight & $35.9 \pm 1.5^{\dagger}$ & $32.5 \pm 1.1$ \\
Body weight gain & $1.9 \pm 0.9$ & $-0.3 \pm 0.9^{* * *}$ \\
Abdominal adipose tissue & & \\
$\quad$ Epididymal & $0.7 \pm 0.2$ & $0.4 \pm 0.1^{* *}$ \\
$\quad$ Perirenal & $0.3 \pm 0.1$ & $0.1 \pm 0.1^{* *}$ \\
Mesenteric & $0.8 \pm 0.2$ & $0.5 \pm 0.1^{*}$ \\
$\quad$ Total & $1.8 \pm 0.5$ & $1.0 \pm 0.2^{* *}$ \\
\hline
\end{tabular}

Data are expressed as the mean $\pm \mathrm{SD}(n=8) .{ }^{\dagger} p<0.01$ vs. initial, ${ }^{* * *} p<0.001$ vs. $\mathrm{CON},{ }^{* *} p<0.01$ vs. $\mathrm{CON},{ }^{*} p<0.05$ vs. CON.

shown in Table 2, hypoxic exposure for 3 weeks significantly reduced body fat mass by approximately $42 \%(p<0.001)$. Furthermore, the relative distribution of fat mass noticeably decreased (Figure 2(b)).

\subsection{Resting Metabolic Rate}

3.3.1. Changes in Energy Expenditure. The energy expenditure for 24 hours was shown the time effects $(p<0.001$; Figure 3(a)), but there was no difference between the groups. However, the sum of energy expenditure over 24 hours was higher in the HYP group than in the CON group by approximately $15 \%(p<001$; Figure 3(b)).

3.3.2. Changes in Respiratory Exchange Rate. Respiratory exchange rate (RER), calculated by the ratio of oxygen uptake and carbon dioxide production, was shown to change over time $(p<0.001$; Figure 3(c)). No differences were found between groups. However, the sum of RER over 24 hours was higher in the HYP group (HYP vs. CON; $0.92 \pm 0.02$ vs. $0.96 \pm 0.02 ; p<0.01$ ) (Figure $3(\mathrm{~d})$ ).

3.3.3. Changes in Carbohydrate and Fat Oxidation. Carbohydrate and fat oxidation were calculated from VCO2 and VO2 values. Carbohydrate oxidation changed over the 24 hours in both groups $(p<0.001$; Figure $4(\mathrm{a}))$. Although no difference was observed between groups, the sum of carbohydrate oxidation over 24 hours was higher in the HYP group by approximately 34\% ( $p<0.001$; Figure $4(\mathrm{~b}))$. Fat oxidation also was shown the time effect in both groups $(p<0.001$; Figure $4(\mathrm{c}))$. No difference was observed between groups. However, the sum of fat oxidation over 24 hours was lower in the HYP group by approximately $29 \%(p<0.01$; Figure $4(d))$.

\subsection{Effects of Hypoxic Exposure on Energy Metabolism} Enzymes in Soleus Muscles. We measured the expression levels of several energy metabolism enzymes by western blot. As shown in Figure 5, no significant difference was observed in the HK2 expression between groups; however, the expression was greater in the HYP group by approximately $21 \%$. The expression of $\mathrm{PDH}$ was significantly increased by approximately $23 \%$ in soleus muscles following hypoxic exposure compared to the CON group $(p<0.05)$. The 


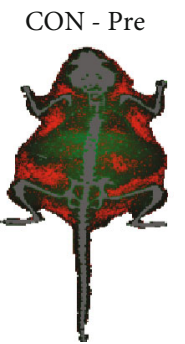

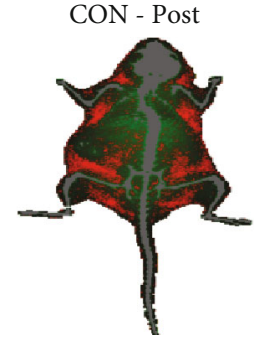

(a)

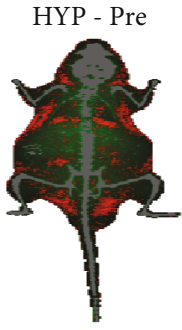

(b)

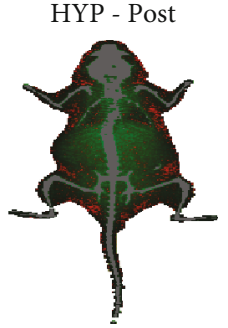

)

FIGURE 2: DXA-scan images of one animal in each treatment group. Radiography of body fat was displayed by two modes according to lowdensity fat (green color) and high-density fat (red color) (a) the CON group (Pre and Post), (b) the HYP group (Pre and Post).

TABLE 2: Change in body weight and adipose tissue weight in the CON and HYP groups according to DXA measurements.

\begin{tabular}{|c|c|c|c|c|c|c|}
\hline & \multicolumn{3}{|c|}{$\mathrm{CON}$} & \multicolumn{3}{|c|}{ HYP } \\
\hline & Initial (g) & Final $(\mathrm{g})$ & Change (\%) & Initial (g) & Final (g) & Change (\%) \\
\hline Fat mass & $3.0 \pm 0.5$ & $2.8 \pm 0.9$ & $-7.8 \%$ & $2.5 \pm 0.3$ & $1.4 \pm 0.1^{* * *}$ & $-42.0 \%$ \\
\hline Fat-free mass & $34.1 \pm 1.5$ & $35.8 \pm 0.8^{*}$ & $5.1 \%$ & $32.6 \pm 1.6$ & $33.8 \pm 1.5^{*}$ & $3.5 \%$ \\
\hline
\end{tabular}

Data are expressed as the mean $\pm \mathrm{SD}(n=8) .{ }^{* * *} p<0.001$ vs. Initial, ${ }^{*} p<0.05$ vs. Initial.

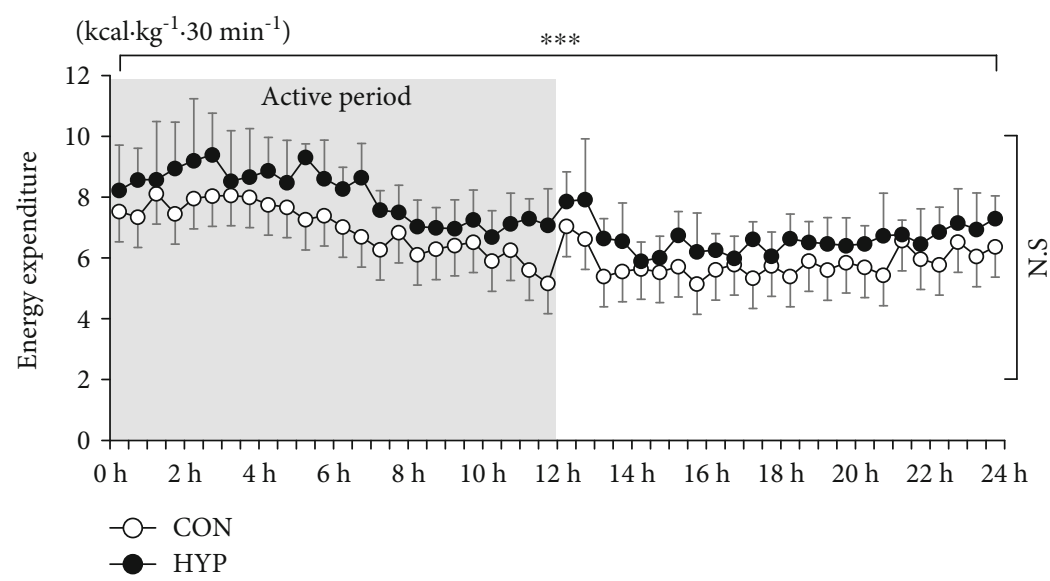

(a)

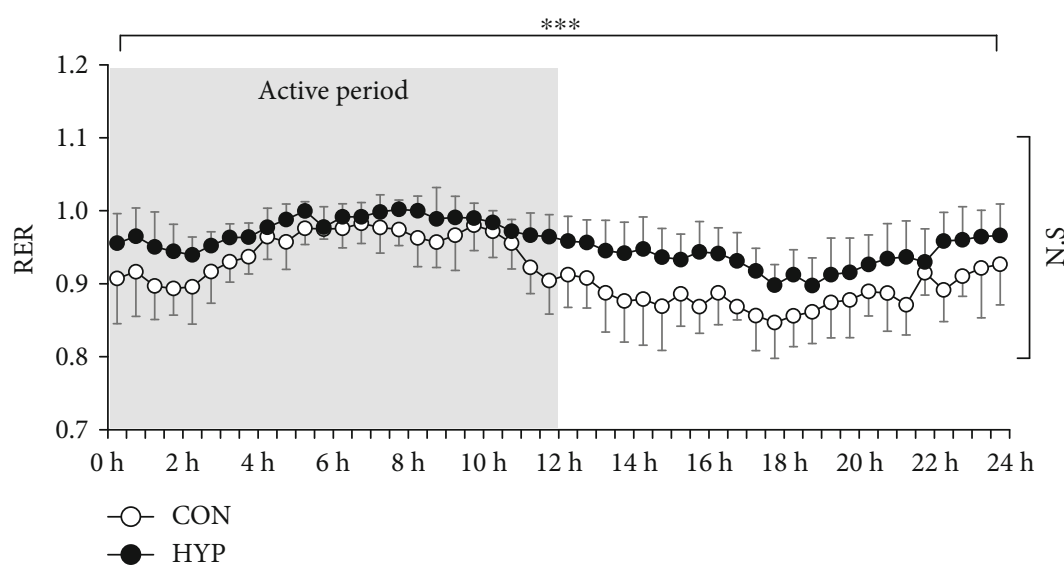

(c)

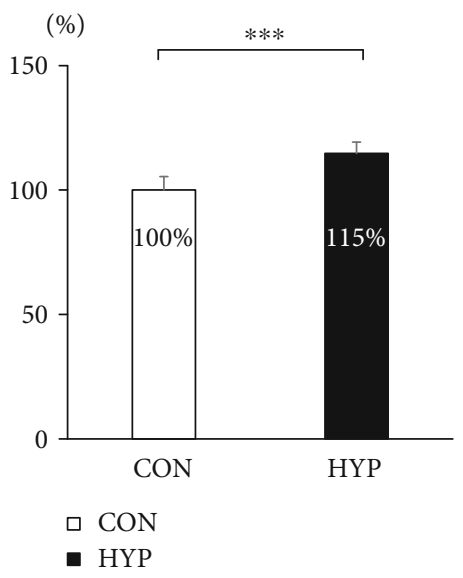

(b)

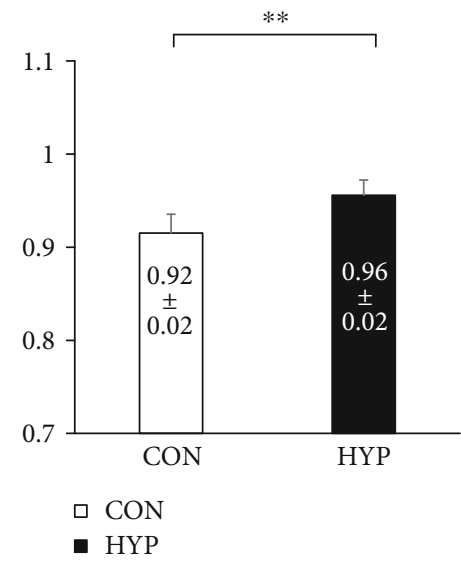

(d)

FIGURE 3: Changes in energy expenditure and respiratory exchange ratio (RER) for 24 hours (a, c) and mean (b, d) in the CON and HYP groups according to RMR measurements. Data are expressed as the mean $\pm \mathrm{SD}(n=8) .{ }^{* * *} p<0.001$, N.S., no significance. 


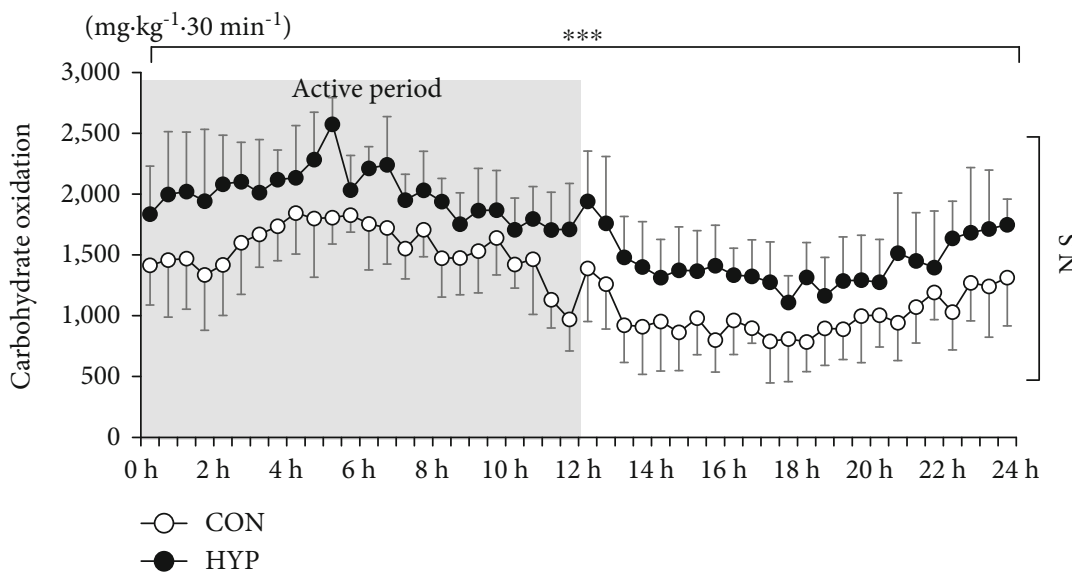

(a)

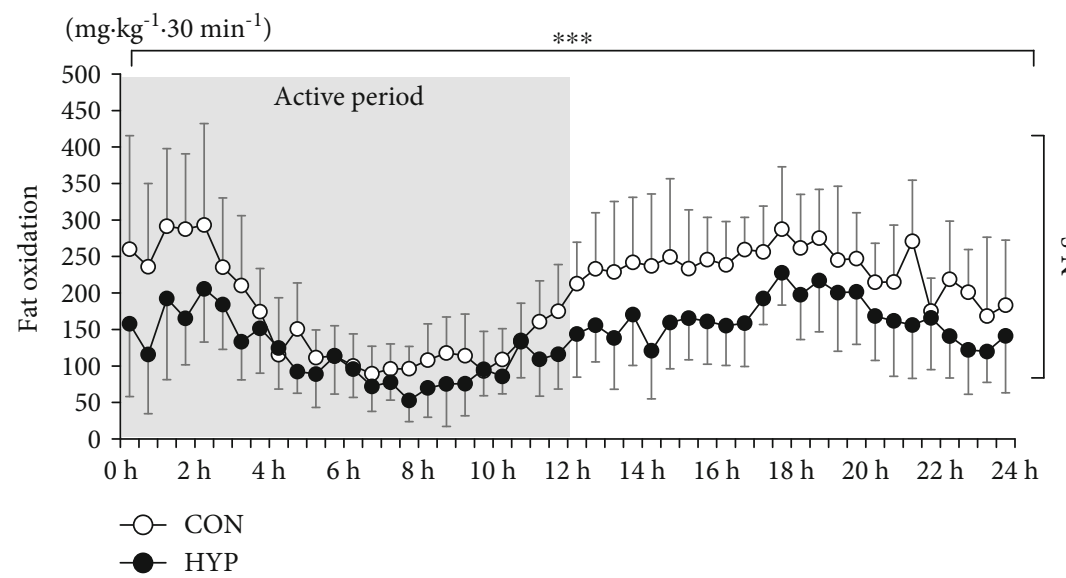

(c)

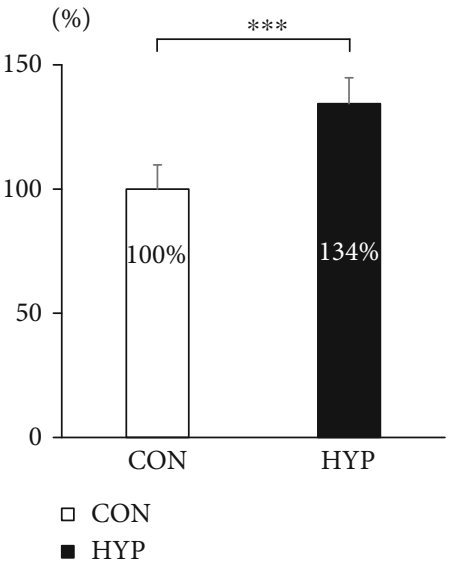

(b)

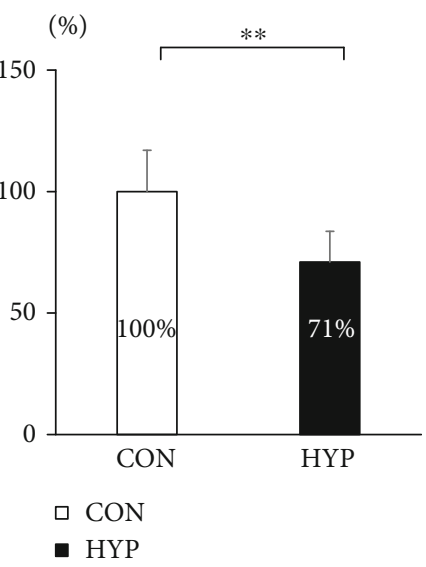

(d)

Figure 4: Changes in carbohydrate and fat oxidation for 24 hours $(a, c)$ and sum $(b, d)$ in the CON and HYP groups according to RMR measurements. Data are expressed as the mean $\pm \operatorname{SD}(n=8) .{ }^{* * *} p<0.001,{ }^{* *} p<0.01$, N.S., no significance.

expression of PDK4 was lower in the HYP group by approximately $10 \%$, but this difference was not statistically significant. Finally, the expression of PGC1 $\alpha$ was higher in the HYP group than in the CON group by approximately $35 \%$ $(p<0.05)$.

\section{Discussion}

We investigated the effects of prolonged (3-week) hypoxic exposure on energy substrate utilization in resting mice by using an open-circuit calorimetry system. We found that carbohydrate oxidation was higher in the HYP group than the CON group. In addition, the resting RER was significantly higher in the HYP group, suggesting that carbohydrate oxidation was enhanced under hypoxic exposure. These results were consistent with Brooks et al. [8], who found that acclimatization to high altitude $(4,300 \mathrm{~m})$ for 3 weeks resulted in enhanced glucose utilization. It was determined that the rate of glucose appearance and disappearance were higher in hypoxic exposure groups.

The capacity for glucose uptake is important to carbohydrate oxidation. Glut4 mediates insulin-stimulated glucose uptake in skeletal muscle. Ying et al. [16] reported that Glut4 protein expression was increased following exposure to hypoxic conditions (fractional inspired $\mathrm{O} 2$ of $9 \pm 0.5 \%$ ) for 30 days. In addition, a previous study reported that highaltitude natives living in mountains above $2,500 \mathrm{~m}$ altitude present lower blood glucose and insulin levels [3]. These reports revealed that hypoxic exposure improves insulin sensitivity. In particular, Wright et al. [17] reported that hypoxia augmented glucose uptake in the skeletal muscle. Since skeletal muscle is the most important tissue for glucose disposal, changes in the metabolic features of the skeletal muscle should affect whole-body insulin regulation [3].

Insulin sensitivity is correlated with body fat mass. Previous studies have reported that insulin-mediated glucose disposal was inversely correlated with body fat mass including total fat and visceral fat $[18,19]$. In the present study, fat mass was significantly decreased in the HYP group by approximately $40 \%$. Additionally, the weight of abdominal adipose tissue in the HYP group was less than that of the control group (HYP vs. CON; $1.0 \pm 0.2$ vs. $1.8 \pm 0.5 \mathrm{~g} ; p<0.01$ ). Based on these results, we assume that the higher capacity for carbohydrate oxidation found in the HYP group of the present study was a result of increased insulin sensitivity mediated by a reduced fat mass. However, the current study did 


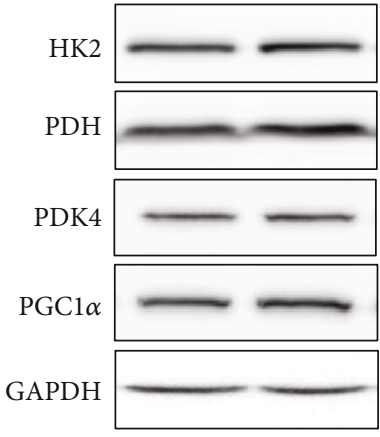

(a)
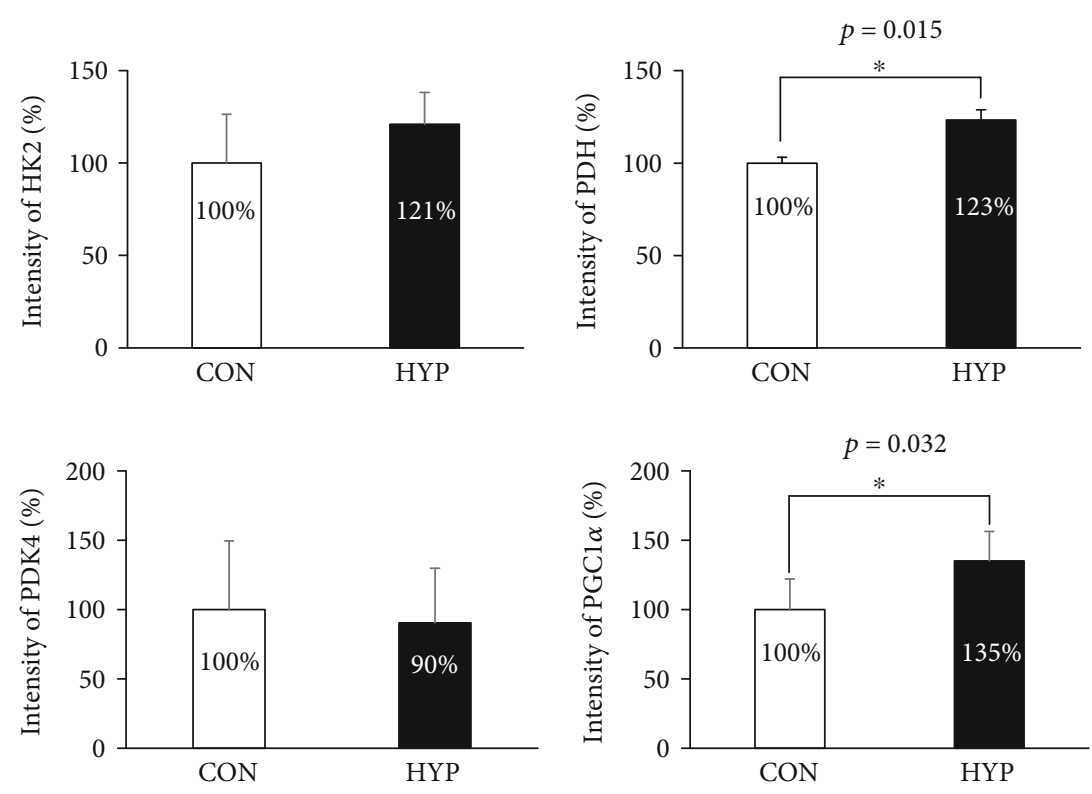

(b)

Figure 5: Effects of hypoxic exposure on the expression of glucose metabolism-related protein variables in the soleus muscle. (a) Soleus muscles were collected immediately after hypoxic exposure, and protein expression was evaluated using western blotting (b) The graphs represent the intensities of the HK2, PDH, PDK4, and PGC1 $\alpha$ bands (\%). Data are expressed as the mean \pm SD $(n=8)$. The band intensity in the hypoxia (HYP) group was set as the control group. ${ }^{*} p<0.05$ vs. the CON group.

not confirm whether hypoxic exposure effects to the insulin sensitivity and glucose tolerance. In addition, because the sample size with 8 mice in each group was a small sample, we acknowledged it as a study limitation. Therefore, future studies are required to elucidate the effect of hypoxia on glycemic control.

In some cases, increased insulin sensitivity was not correlated with the activity of glucose transporters following exposure to hypoxic conditions (fractional inspired $\mathrm{O} 2$ of $10 \%$ ) compared with normoxic conditions [3]. Therefore, it was necessary to determine whether the proteins involved in the carbohydrate metabolic mechanism were upregulated under hypoxic conditions. Our findings support the hypothesis that hypoxic exposure could promote carbohydrate oxidation by increasing the expression level of enzymes related to carbohydrate metabolism. We measured the levels of HK2, PDH, $\mathrm{PDK} 4$, and PGC1 $\alpha$ in mice after exposure to hypoxic conditions. Hexokinases phosphorylate glucose to glucose-6-phosphate, which is the first step in carbohydrate metabolism pathways. HK2 gene expression can be induced by factors such as insulin, hypoxia, and exercise. HK2 can effectively prevent glucose from leaving the cell and, thus, commits glucose to the energy metabolism pathway [20]. No significant difference was observed in HK2 expression between HYP and CON groups; however, the expression was greater in the HYP group by approximately $21 \%$. Hypoxic exposure significantly increased the expression of the PDH protein by approximately $23 \%$. The increase in $\mathrm{PDH}$ suggests a greater capacity for glucose utilization. Pyruvate dehydrogenase kinase 4 (PDK4) inhibits PDH via phosphorylation. Although our results did not show significant differences in PDK4 between HYP and CON groups, it was lower in the
HYP group compared with the CON group by approximately $10 \%$. Accordingly, it was demonstrated that the enhanced expression of PDH and HK2 is correlated with increases in carbohydrate oxidation under hypoxic conditions.

We also found that energy expenditure following hypoxic exposure was higher than normoxic conditions. Fat oxidation was lower in the HYP group than the CON group. However, there was also a significant reduction in fat mass in the HYP group of approximately $42 \%$ (Table 2). Therefore, the change in fat mass assumed to have resulted from increased energy expenditure itself. In human studies, altitude has been shown to have an important impact on energy balance and body composition. Butterfield et al. [21] has reported a loss in body mass during altitude sojourns (altitude $4,300 \mathrm{~m}$ for 1 month) and have found that basal metabolic rate increases at high altitude. Mawson et al. [22] demonstrated that altitude exposure at 4,300 m elevates basal metabolic rate compared with rates at sea level. In another study, during altitude exposures between $3,500 \mathrm{~m}$ and 5,000 $\mathrm{m}$, RMR was increased by $10 \%$ and $28 \%$, respectively [23]. In the case of animals, highland deer mice expend significantly more energy per day than mice from a lowland population, which would require a high rate of $\mathrm{O} 2$ and substrate consumption [6]. Highland deer mice may be expected to rely on carbohydrates, as carbohydrates are metabolized with superior yield of ATP per mole $\mathrm{O} 2$ consumed. In order to compensate for decreased availability of $\mathrm{O} 2$, oxidative muscles (i.e., soleus muscle) have increased mitochondrial function. A prominent role of mitochondria is to produce the energy by consuming oxygen. Ferri et al. [24] assessed the respiratory parameters of the mitochondrial population of soleus muscle fiber and found that mitochondrial respiration was 
significantly higher following hypoxic exposure (fractional inspired $\mathrm{O} 2$ of $10 \%$ for 4 weeks) compared with normoxic condition for all respiration states. According to these results, we hypothesize that the increased energy expenditure results from enhancing mitochondrial activation in response to decreased availability of $\mathrm{O} 2$.

Although an investigation of mitochondrial function was not conducted in the present study, we did assess the protein expression of PGC1 $\alpha$. PGC1 $\alpha$ is the key regulator of mitochondrial biogenesis. O'Hagan et al. [25] reported that PGC-1 $\alpha$ upregulation in skeletal muscle cells led to increased oxygen consumption secondary to increased mitochondrial mass. In our study, the protein expression of PGC1 $\alpha$ was approximately $35 \%$ higher in mice exposed to hypoxic conditions (Figure 5). Based on these results, we concluded that hypoxic exposure could augment energy expenditure via PGC- $1 \alpha$ upregulation.

\section{Conclusions}

Prolonged exposure to hypoxia for 3 weeks, at $12 \%$ oxygen ( $\approx$ altitude $4,300 \mathrm{~m}$ ) augmented total energy expenditure and carbohydrate oxidation. Moreover, body fat mass was decreased in the HYP group. From a clinical perspective, exposure to hypoxia may be a realistic method for treating type 2 diabetes. However, the current study did not confirm whether hypoxic exposure effects to the insulin sensitivity and glucose tolerance. Therefore, future studies are required to elucidate the molecular variables of glycemic control.

\section{Data Availability}

The data used to support the findings of this study are available from the corresponding author upon request.

\section{Conflicts of Interest}

The authors declare that there are no conflicts of interest regarding the publication of this paper.

\section{Authors' Contributions}

Kiwon Lim and Jisu Kim contributed to the design of all experiments and interpreted the results of the data. Jisu Kim and Hun-Young Park interpreted the results of the data and revised the paper. Yeram Park collected the data, undertook the statistical analyses, and wrote the manuscript. Deunsol Hwang carried out the studies and provided technical support. All of the authors read and approved the final manuscript.

\section{Acknowledgments}

This study was supported by a grant (NRF-2015M3C1B 1019479) from the National Research Foundation funded by the Korean Government.

\section{References}

[1] H. Y. Park, J. S. Kim, and K. W. Lim, "Exercise physiology basis and necessity of hypoxic training to improve exercise performance in elite athletes," Korean Journal of Sport Science, vol. 29, no. 4, pp. 737-752, 2018.

[2] J. B. West, "The physiologic basis of high-altitude diseases," Annals of Internal Medicine, vol. 141, no. 10, pp. 789-800, 2004.

[3] J. L. Gamboa, M. L. Garcia-Cazarin, and F. H. Andrade, "Chronic hypoxia increases insulin-stimulated glucose uptake in mouse soleus muscle," American Journal of Physiology-Regulatory, Integrative and Comparative Physiology, vol. 300, no. 1, pp. R85-R91, 2011.

[4] N. E. Hill, K. Deighton, J. Matu et al., "Continuous glucose monitoring at high altitude-effects on glucose homeostasis," Medicine and Science in Sports and Exercise, vol. 50, no. 8, pp. 1679-1686, 2018.

[5] O. O. Woolcott, O. A. Castillo, C. Gutierrez, R. M. Elashoff, D. Stefanovski, and R. N. Bergman, "Inverse association between diabetes and altitude: a cross-sectional study in the adult population of the United States," Obesity, vol. 22, no. 9, pp. 2080-2090, 2014.

[6] J. P. Hayes and C. S. O'Connor, "Natural selection on thermogenic capacity of high-altitude deer mice," Evolution, vol. 53, no. 4, pp. 1280-1287, 1999.

[7] M. P. Schippers, O. Ramirez, M. Arana, P. Pinedo-Bernal, and G. B. McClelland, "Increase in carbohydrate utilization in high-altitude andean mice," Current Biology, vol. 22, no. 24, pp. 2350-2354, 2012.

[8] G. A. Brooks, G. E. Butterfield, R. R. Wolfe et al., "Increased dependence on blood glucose after acclimatization to 4,300 m," Journal of Applied Physiology, vol. 70, no. 2, pp. 919-927, 1991.

[9] O. O. Woolcott, M. Ader, and R. N. Bergman, "Glucose homeostasis during short-term and prolonged exposure to high altitudes," Endocrine Reviews, vol. 36, no. 2, pp. 149173, 2015.

[10] A. C. Roberts, G. E. Butterfield, A. Cymerman, J. T. Reeves, E. E. Wolfel, and G. A. Brooks, "Acclimatization to 4,300-m altitude decreases reliance on fat as a substrate," Journal of Applied Physiology, vol. 81, no. 4, pp. 1762-1771, 1996.

[11] K. Goto, T. Morishima, K. Kurobe, Z. Huang, and F. Ogita, "Augmented carbohydrate oxidation under moderate hypobaric hypoxia equivalent to simulated altitude of $2500 \mathrm{~m}$," The Tohoku Journal of Experimental Medicine, vol. 236, no. 3, pp. 163-168, 2015.

[12] J. Yeu, H.-J. Ko, D. Kim et al., "Evaluation of iNSiGHT VET DXA (Dual-Energy X-ray Absorptiometry) for assessing body composition in obese rats fed with high fat diet: a follow-up study of diet induced obesity model for 8 weeks," Laboratory Animal Research, vol. 35, no. 1, 2019.

[13] J. Kim, H. Hwang, H. Yun et al., "Silk peptide intake increases fat oxidation at rest in exercised mice," Journal of Nutritional Science and Vitaminology, vol. 59, no. 3, pp. 250-255, 2013.

[14] J. Kim, Y. Jeon, H. Hwang, H. Suh, and K. Lim, "Effects of oral caffeine and capsaicin administration on energy expenditure and energy substrates utilization in resting rats," The Journal of Exercise Nutrition and Biochemistry, vol. 15, no. 4, pp. 183-189, 2011.

[15] N. Chung, J. Park, and K. Lim, "The effects of exercise and cold exposure on mitochondrial biogenesis in skeletal muscle and 
white adipose tissue," Journal of Exercise Nutrition \& Biochemistry, vol. 21, no. 2, pp. 39-47, 2017.

[16] Y. Xia, J. B. Warshaw, and G. G. Haddad, "Effect of chronic hypoxia on glucose transporters in heart and skeletal muscle of immature and adult rats," American Journal of PhysiologyRegulatory, Integrative and Comparative Physiology, vol. 273, no. 5, pp. R1734-R1741, 1997.

[17] D. C. Wright, P. C. Geiger, J. O. Holloszy, and D.-H. Han, "Contraction- and hypoxia-stimulated glucose transport is mediated by a $\mathrm{Ca}^{2+}{ }_{-}$-dependent mechanism in slow-twitch rat soleus muscle," American Journal of PhysiologyEndocrinology and Metabolism, vol. 288, no. 6, pp. E1062E1066, 2005.

[18] A. J. Ruiz-Alcaraz, C. Lipina, J. R. Petrie et al., "Obesityinduced insulin resistance in human skeletal muscle is characterised by defective activation of p42/p44 MAP kinase," PLoS One, vol. 8, no. 2, article e56928, 2013.

[19] A. Raji, E. W. Seely, R. A. Arky, and D. C. Simonson, "Body fat distribution and insulin resistance in healthy Asian Indians and Caucasians," The Journal of Clinical Endocrinology \& Metabolism, vol. 86, no. 11, pp. 5366-5371, 2001.

[20] R. L. Printz, S. Koch, L. R. Potter et al., "Hexokinase II mRNA and gene structure, regulation by insulin, and evolution," Journal of Biological Chemistry, vol. 268, no. 7, pp. 5209-5219, 1993.

[21] G. E. Butterfield, J. Gates, S. Fleming, G. A. Brooks, J. R. Sutton, and J. T. Reeves, "Increased energy intake minimizes weight loss in men at high altitude," Journal of Applied Physiology, vol. 72, no. 5, pp. 1741-1748, 1992.

[22] J. T. Mawson, B. Braun, P. B. Rock, L. G. Moore, R. Mazzeo, and G. E. Butterfield, "Women at altitude: energy requirement at 4,300 m," Journal of Applied Physiology, vol. 88, no. 1, pp. 272-281, 2000.

[23] B. Kayser, "Nutrition and energetics of exercise at altitude. Theory and possible practical implications," Sports Medicine, vol. 17, no. 5, pp. 309-323, 1994.

[24] A. Ferri, A. Panariti, G. Miserocchi et al., "Tissue specificity of mitochondrial adaptations in rats after 4 weeks of normobaric hypoxia," European Journal of Applied Physiology, vol. 118, no. 8, pp. 1641-1652, 2018.

[25] K. A. O'Hagan, S. Cocchiglia, A. V. Zhdanov et al., "PGC-1 is coupled to HIF-1 -dependent gene expression by increasing mitochondrial oxygen consumption in skeletal muscle cells," Proceedings of the National Academy of Sciences, vol. 106, no. 7, pp. 2188-2193, 2009. 